\title{
Antineutrophil cytoplasmic antibody-associated crescentic glomerulonephritis with membranous nephropathy treated using thiamazole
}

\author{
Tomoko Kakita $\cdot$ Katsuyuki Nagatoya $\cdot$ Hiroki Takimoto $\cdot$ \\ Hirohisa Matsuda - Tatsuhiko Mori · Ayako Kawaguchi • \\ Toru Inoue
}

Received: 5 September 2012 / Accepted: 7 January 2013/Published online: 12 March 2013

(C) Japanese Society of Nephrology 2013

\begin{abstract}
A 21-year-old woman with nephrotic syndrome was referred to our hospital. She had congenital diaphragmatic hernia, hypoxic ischemic encephalopathy, and mental retardation, and had been treated for hyperthyroidism with thiamazole in another hospital. Serum creatinine was $37.8 \mu \mathrm{mol} / \mathrm{L}$ and antineutrophil cytoplasmic antibody against myeloperoxidase (MPO-ANCA) was 39 EU. Urinalyses were $3+$ for proteins and $3+$ for occult blood. A renal biopsy was performed. An examination using light microscopy (LM) revealed necrotizing glomerulonephritis with crescent formation. Immunofluorescence microscopy showed granular staining with immunoglobulin $G$ and complement component 3 along the capillary walls. Electron microscopy (EM) disclosed subepithelial dense deposits. A renal biopsy suggested necrotizing glomerulonephritis with membranous nephropathy (MN) in stages I or II. Since many cases of drug-induced ANCA-associated glomerulonephritis (AAG) have been reported, we stopped thiamazole and treated with corticosteroid. The MPOANCA titer became negative 49 days after the initiation of
\end{abstract}

T. Kakita $\cdot$ K. Nagatoya $(\bowtie) \cdot$ H. Takimoto $\cdot$ H. Matsuda

T. Mori · A. Kawaguchi - T. Inoue

Department of Nephrology, Osaka Medical College Hospital,

2-7 Daigaku-machi, Takatsuki, Osaka 569-8686, Japan

e-mail: katsuyuki@poh.osaka-med.ac.jp

Present Address:

A. Kawaguchi

Takamatsu Medical Clinic, 76-1 Urada,

Kaizuka, Osaka 597-0061, Japan

Present Address:

T. Inoue

Department of Internal Medicine, General Hospital

Higashikouri, 1-24-34 Higashikouri, Hirakata,

Osaka 573-0075, Japan treatment. Two years after the first treatment, the MPOANCA titer became elevated again and was $82 \mathrm{EU}$. The patient was administered cyclophosphamide and prednisone. However, the MPO-ANCA titer did not decrease. A renal biopsy was performed again 3 years after the first renal biopsy. LM revealed no crescentic formation but demonstrated spike formations along the glomerular basement membrane. EM also disclosed subepithelial dense deposits, but less than the first biopsy. The renal biopsy suggested MN in stages II or III. AAG was regarded as inactive after corticosteroid treatment. Therefore, ciclosporin administration was started. In conclusion, we experienced a rare case of AAG complicated with MN. The histopathologic results showed that immunosuppressive therapy seemed to be effective in treating crescentic glomerulonephritis; furthermore, it reduced proteinuria but could not reduce the MPO-ANCA titer.

Keywords ANCA-associated crescentic glomerulonephritis - Membranous nephropathy · Antithyroid drug

\section{Introduction}

Antineutrophil cytoplasmic antibody (ANCA)-associated crescentic glomerulonephritis is the most common cause of crescentic glomerulonephritis. It is commonly accompanied with hematuria and proteinuria, but the degree of proteinuria is generally in the subnephrotic range $[1,2]$. Sometimes, hyperthyroidism patients treated with an antithyroid drug may test positive for antineutrophil cytoplasmic antibody against myeloperoxidase (MPO-ANCA) accompanied with hematuria. There is no standard therapy for these patients [3]. However, generally, administration of the 
antithyroid drug is stopped. There has been a report on a comparison between drug-induced and idiopathic ANCA vasculitis, where drug-induced ANCA-associated glomerulonephritis (AAG) had a more moderate course and immunosuppressive therapy was not necessary in some cases [4]. Membranous nephropathy (MN) is a pathologic diagnosis characterized by a spectrum of change in the glomerular basement membrane. It is the most common cause of nephrotic syndrome in nondiabetic adults and is associated with a variety of diseases such as hepatitis B, autoimmune diseases, thyroiditis, and malignancies. However, it is very rare for MN to occur secondary to AAG [5]. The present study reports a case of AAG with MN treated using thiamazole.

\section{Case report}

A 21-year-old woman with nephrotic syndrome was referred to our hospital. She had congenital diaphragmatic hernia, hypoxic ischemic encephalopathy, and mental retardation, and had been successfully treated for hyperthyroidism with thiamazole in another hospital for 5 years. She had eyelid and lower limb edema 1 month before admission. On admission, laboratory tests showed hemoglobin $127 \mathrm{~g} / \mathrm{L}$, total protein $49 \mathrm{~g} / \mathrm{L}$, albumin $19 \mathrm{~g} / \mathrm{L}$, serum creatinine (Cre) $45.8 \mu \mathrm{mol} / \mathrm{L}$, blood urea nitrogen $4.28 \mathrm{mmol} / \mathrm{L}, \mathrm{C}$-reactive protein (CRP) $48.5 \mathrm{nmol} / \mathrm{L}$, total cholesterol $7.95 \mathrm{nmol} / \mathrm{L}$, triglyceride $2.88 \mathrm{mmol} / \mathrm{L}$, and high-density lipoprotein cholesterol $1.11 \mathrm{nmol} / \mathrm{L}$. Free thyroxine 4 was $14.8 \mathrm{pmol} / \mathrm{L}$ and thyroid-stimulating hormone level was $10.7 \mathrm{mIU} / \mathrm{L}$. Levels of immunoglobulin $\mathrm{G}$ $(\operatorname{IgG}), \mathrm{A}(\operatorname{Ig} \mathrm{A})$, and $\mathrm{M}(\operatorname{IgM})$ were $8.74 \mathrm{~g} / \mathrm{L}, 3110 \mathrm{mg} / \mathrm{L}$, and $1210 \mathrm{mg} / \mathrm{L}$, respectively. Levels of complement component 3 (C3) and complement component 4 were $1.64 \mathrm{~g} / \mathrm{L}$ (normal range $0.65-1.35 \mathrm{~g} / \mathrm{L}$ ) and $0.366 \mathrm{~g} / \mathrm{L}$ (normal range $0.130-0.350 \mathrm{~g} / \mathrm{L}$ ), respectively. The antinuclear antibody tested negative and the levels of ANCA against myeloperoxidase (MPO-ANCA) was $79 \mathrm{EU}$ (normal range $<20 \mathrm{EU}$ ). ANCA against proteinase 3, complement component 1q (C1q), or anti-double-stranded DNA antibody were negative. Urinalyses were $3+$ for occult blood and $3+$ for proteins, $6.4 \mathrm{~g} / \mathrm{g}$ Cre. AAG was suspected, although it rarely has the complication of nephrotic syndrome [1]. Therefore, we performed a renal biopsy under general anesthesia due to the condition of mental retardation being present. Light microscopy (LM) showed 7 cellular crescents with necrosis and 4 fibrocellular crescents out of 32 glomeruli. Immunofluorescence microscopy (IF) showed diffuse granular staining with $\operatorname{IgG}$ and $\mathrm{C} 3$ along the glomerular capillary walls (GCW). IgA, IgM, and C1q were negative. Electron microscopy (EM) disclosed subepithelial and mesangial electron-dense deposits (EDDs) (Fig. 1).
The overall results of the renal biopsy suggested necrotizing glomerulonephritis with $\mathrm{MN}$ in stages I or II.

The clinical course is shown in Fig. 2. Since antithyroid drug-induced AAG has been previously reported, thiamazole administration was stopped. After stopping
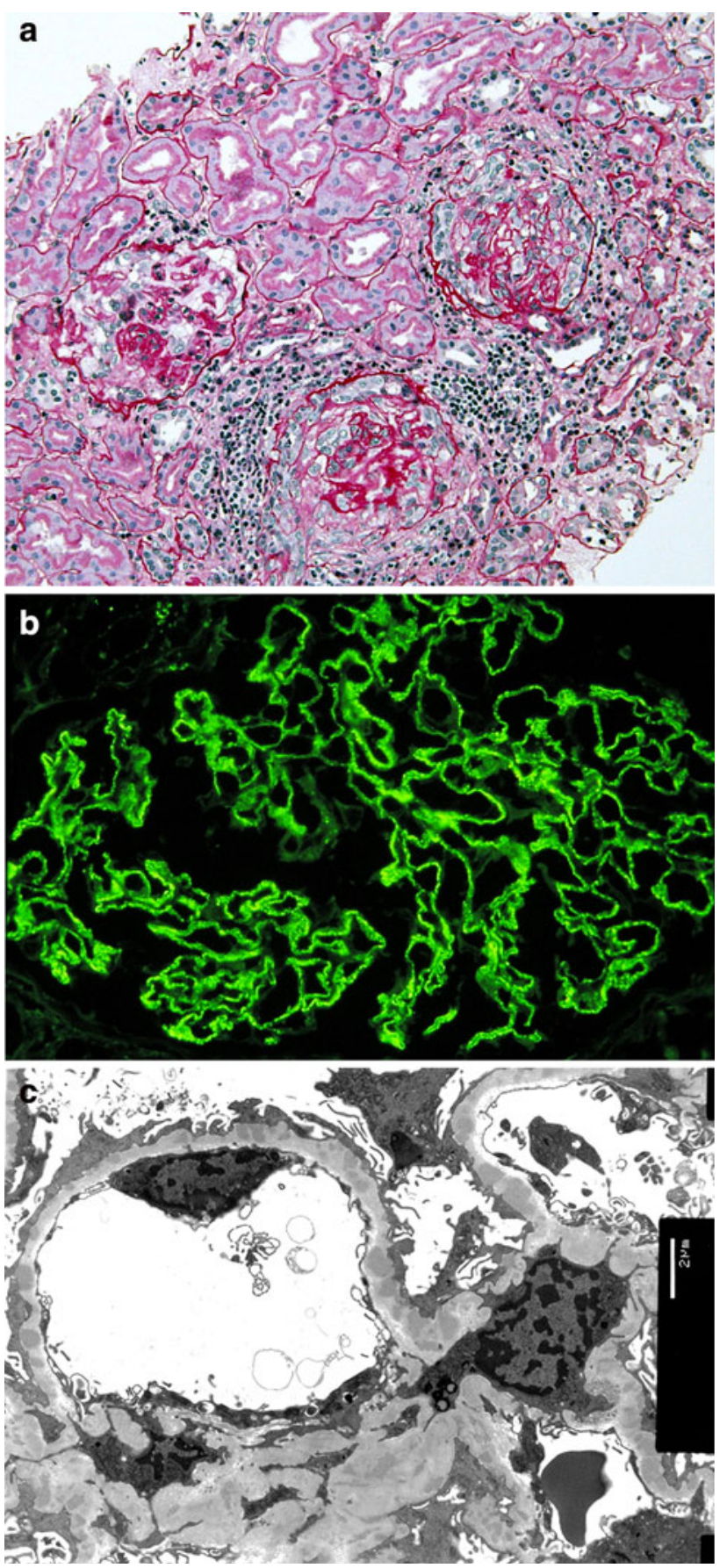

Fig. 1 First renal biopsy samples. a Periodic acid Schiff (PAS) staining $\times 200$ showing cellular crescents. b Immunofluorescence microscopy (IF) of IgG showing fine granular IgG deposition along the glomerular capillary walls. c Electron microscopy (EM) showing dense deposits in the subepithelial space of the glomerular basement membrane 
Fig. 2 Clinical course. Month 0 is the day of first admission. $C Y$ cyclophosphamide, $P S L$ prednisolone, mPSL methylprednisolone, AZT azathioprine

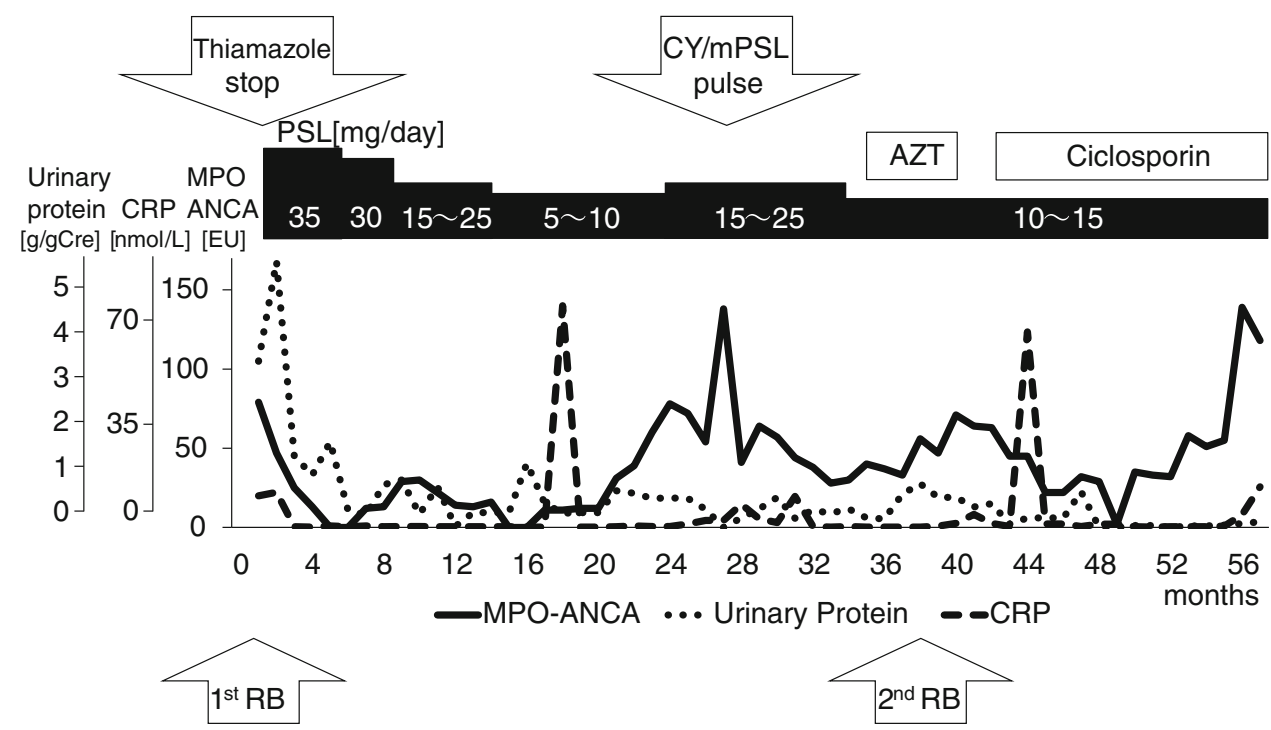

thiamazole, the subject's thyroid hormones were stable and the administration of prednisolone was begun. The MPOANCA titer became very low 49 days after the initiation of treatment. Urinary protein level lowered to $1.0 \mathrm{~g} / \mathrm{g}$ Cre, her edema disappeared, and prednisolone was tapered. Six months after starting steroid therapy, the MPO-ANCA titer had remained low at around $20 \mathrm{EU}$ with prednisolone administered at $10 \mathrm{mg} /$ day.

Two years after the first treatment, the MPO-ANCA titer became elevated again to $82 \mathrm{EU}$. The subject had no symptoms and her CRP was negative. Prednisolone was, once again, increased to $25 \mathrm{mg} /$ day. However, it was reduced again and the administration of intravenous pulse cyclophosphamide was started due to the side effects of prednisolone, such as insomnia and appetite loss. Since the MPO-ANCA titer continued to be elevated, intravenous pulse methylprednisolone was administered. After that treatment, the MPO-ANCA titer fell once again. However, it fluctuated again. Therefore, azathioprine was temporarily administered but discontinued because of liver damage. We performed a renal biopsy again 3 years after the first renal biopsy to reevaluate her renal condition and to reconsider her therapeutic strategy. LM revealed no crescentic formation or necrosis, but demonstrated spike formations along the glomerular basement membrane. EM also disclosed subepithelial EDDs, but the amount was less than that of the first biopsy (Fig. 3). The second renal biopsy suggested MN in stages II or III. Since AAG appeared to have become inactive by virtue of the treatment, mild immunosuppressive therapy was thought to be desirable and ciclosporin administration was started. Although her MPO-ANCA titer has remained relatively high, she has had

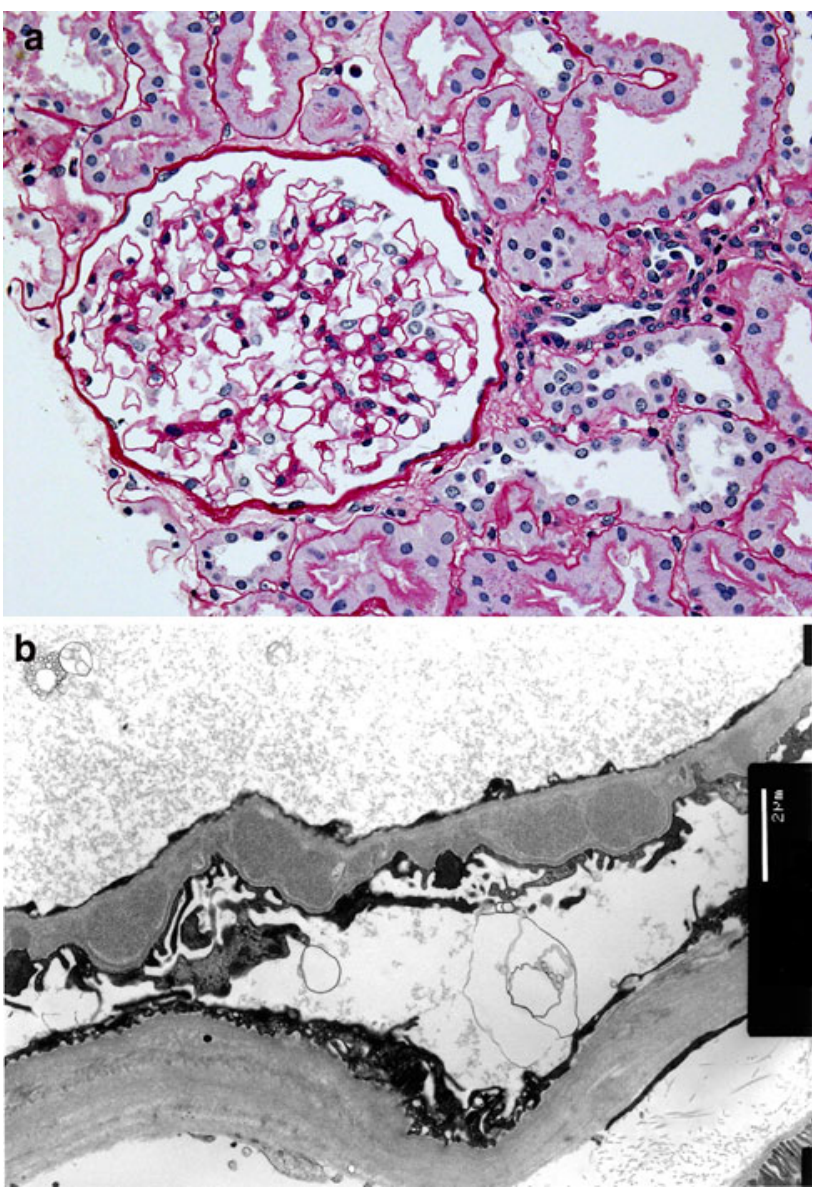

Fig. 3 Second renal biopsy samples. a PAS staining $\times 400$ showing no crescentic formation. b Electron microscopy (EM) showing dense deposits in the subepithelial space of the glomerular basement membrane 
no symptoms and her CRP level has remained negligible at the time of writing of this article.

\section{Discussion}

AAG rarely has the complication of nephrotic syndrome [1]. There are some reports of AAG with $\mathrm{MN}$ [6-8]. In a case reported by Matsumoto et al., there was MPO deposition along the GCW in the first biopsy, which disappeared in the second biopsy and was accompanied by a decreased MPO-ANCA titer after treatment. They concluded that MPO and MPO-ANCA might have been responsible for the IgG immune depositions along the GCW in their patient [6]. In the present case, although MPO immunofluorescence was not performed, MPO-ANCA seemed to have no relationship with IgG immune depositions along the GCW because MPO-ANCA remained high after IgG deposition disappeared with steroid therapy. IgG deposition along the basement membrane in the present case was hypothesized to be due to unknown deposits produced by MN.

It was not elucidated as to whether the pathogenesis of ANCA-associated vasculitis in the present patient was drug induced. Drug-induced ANCA-associated vasculitis usually presents with skin lesions and fewer renal lesions than that of idiopathic AAG [3, 4]. The present patient had no skin lesions, but had renal involvement. After stopping thiamazole, the MPO-ANCA titer was still elevated. Most reported cases of antithyroid drug-induced AAG have been caused by propylthiouracil, and only a few by thiamazole $[9,10]$. In this respect, the present case is less likely to be drug-induced AAG.

Viewing the clinical course retrospectively, the crescentic glomerulonephritis did not necessarily correlate with the MPO-ANCA titer in the present case. However, it has been reported that an elevated ANCA titer could help predict relapses and that a preemptive increase in immunosuppression reduces the risk of relapses [11]. Therefore, sufficient immunosuppressive therapy was administered on the basis of the ANCA titer until the second biopsy was performed and either deterioration of renal function or new involvement of ANCA vasculitis in other organs was not observed. However, long-term immunosuppressive therapy causes various adverse effects, some of which (e.g., opportunistic infections) are fatal. There is still no consensus with respect to striking a balance between sufficient immunosuppression and the prevention of adverse effects.

As mentioned in the "Introduction", $\mathrm{MN}$ is associated with diverse morbidities [5]. However, except for hyperthyroidism, no concurrent disease was detected in the current patient. Because hyperthyroidism is not generally associated with $\mathrm{MN}$, the cause of $\mathrm{MN}$ in this case seemed to be idiopathic or unknown ANCA-associated origin.
Although EDDs in the mesangium have been reported to be observed in secondary MN like lupus type $\mathrm{V}$ [12], it may not be exceptional in MN complicated with AAG, even though it is not secondary. In fact, EDDs in the mesangium were observed in 6 of 13 cases in a case series [7] and also in another case report [6]. Although EDDs in the mesangium are frequently observed in lupus nephritis without membranous lesions, they are not generally observed in AAG. Therefore, EDDs are not simply due to AAG. The reason why EDDs in the mesangium are frequently observed in MN complicated with AAG has not yet been elucidated. $\mathrm{MN}$ in young adults is, itself, relatively rare, and MN complicated with AAG in such cases is extremely rare. The key pathophysiological relationship between these 2 morbidities remains to be elucidated.

In conclusion, we report a rare case of AAG complicated with MN. Immunosuppressive therapy seemed to be effective in treating crescentic glomerulonephritis histopathologically and in reducing proteinuria, while it was refractory to reducing the MPO-ANCA titer.

Conflict of interest All of the authors have declared that they have no competing interests.

\section{References}

1. Jennette JC, Thomas DB. Pauci-immune and antineutrophil cytoplasmic autoantibody-mediated crescentic glomerulonephritis and vasculitis. In: Jennette JC, Olson JL, Schwartz MM, Silva FG, editors. Heptinstall's pathology of the kidney. 6th ed. Philadelphia: Lippincott Williams \& Wilkins; 2007. p. 644-73.

2. Levy J, Pusey CD. Crescentic glomerulonephritis. In: Davison AMA, editor. Oxford textbook of clinical nephrology. 3rd ed. Oxford: Oxford University Press; 2005. p. 559-77.

3. Bonaci-Nikolic B, Nikolic MM, Andrejevic S, Zoric S, Bukilica M. Antineutrophil cytoplasmic antibody (ANCA)-associated autoimmune diseases induced by antithyroid drugs: comparison with idiopathic ANCA vasculitides. Arthritis Res Ther. 2005;7: R1072-81.

4. Radić M, Martinović Kaliterna D, Radić J. Drug-induced vasculitis: a clinical and pathological review. Neth J Med. 2012;70: 12-7.

5. Schwartz MM. Membranous glomerulonephritis. In: Jennette JC, Olson JL, Schwartz MM, Silva FG, editors. Heptinstall's pathology of the kidney. 6th ed. Philadelphia: Lippincott Williams \& Wilkins; 2007. p. 206-51.

6. Matsumoto K, Honda H, Shibata T, Sanada D, Wada Y, Ashikaga E, Kuroki A, Kitazawa K, Akizawa T. MPO-ANCA crescentic glomerulonephritis complicated by membranous nephropathy: MPO demonstrated in epimembranous deposits. NDT Plus. 2009; 2:461-5.

7. Nasr SH, Said SM, Valeri AM, Stokes MB, Masani NN, D’Agati VD, Markowitz GS. Membranous glomerulonephritis with ANCA-associated necrotizing and crescentic glomerulonephritis. Clin J Am Soc Nephrol. 2009;4:299-308.

8. Sekine S, Yumura W, Tanaka Y, Suganuma S, Onuki T, Uchida K, Kawashima A, Honda K, Nitta K, Nihei H. An elderly case of ANCA-positive membranous nephropathy. Nihon Jinzo Gakkai Shi. 2002;44:44-9. 
9. Wada N, Mukai M, Kohno M, Notoya A, Ito T, Yoshioka N. Prevalence of serum anti-myeloperoxidase antineutrophil cytoplasmic antibodies (MPO-ANCA) in patients with Graves' disease treated with propylthiouracil and thiamazole. Endocr J. 2002;49:329-34.

10. Bauer AJ. Approach to the pediatric patient with Graves' disease: when is definitive therapy warranted? J Clin Endocrinol Metab. 2011;96:580-8.
11. Han WK, Choi HK, Roth RM, McCluskey RT, Niles JL. Serial ANCA titers: useful tool for prevention of relapses in ANCAassociated vasculitis. Kidney Int. 2003;63:1079-85.

12. Jennette JC, Iskandar SS, Dalldorf FG. Pathologic differentiation between lupus and nonlupus membranous glomerulopathy. Kidney Int. 1983;24:377-85. 